\title{
Prospects of CKM parameter measurements at Belle
} II

Ilya Komarov*†

INFN Trieste and SNSF

E-mail: ilya.komarovets.infn.it

The Belle II experiment is a substantial upgrade of the Belle detector that will operate at the SuperKEKB energy-asymmetric $e^{+} e^{-}$collider. Belle II is preparing to start recording collisions at the $\Upsilon(4 S)$ energy in 2018 and collect $50 \mathrm{ab}^{-1}$ of data by 2025, a factor of 50 more than the Belle experiment. We report prospects for measuring the Cabibbo-Kobayashi-Maskawa angle $\phi_{3}(\gamma)$ and observables in semileptonic $B$ meson decays associated with the unitary matrix element $V_{u b}$.

The European Physical Society Conference on High Energy Physics

5-12 July, 2017

Venice

* Speaker.

${ }^{\dagger}$ on behalf of Belle II collaboration 


\section{Introduction}

Belle II is $4 \pi$ solid angle detector that is being installed at the SuperKEKB $e^{+} e^{-}$energyasymmetric collider in Tsukuba, Japan. Belle II inherits the principal design of its ancestor, Belle, but upgrades of all of the subsystems will yield improved performance. Of special interest are the expected factor of two improvement in vertex resolution offered by the novel pixel detector, increased $K_{s}^{0}$ detection efficiency due to an enlarged vertex detector and improved $\pi^{0}$ reconstruction efficiency provided by an optimized tuning of the electromagnetic calorimeter [1]. Belle II is expected to be comissioned with data at the $\Upsilon(4 S)$ energy in 2018 , aiming to collect $50 \mathrm{ab}^{-1}$ of data by 2025 .

The chief goal of the Belle II experiment is to test the Standard Model and search for non Standard Model physics using precision measurement at the intensity frontier. A significant component of this will be accomplished through probes of unitarity of the Cabibbo-Kobayashi-Maskawa (CKM) triangle [2,3], which requires independent measurements of the triangle parameters. Some CKM parameters $\left(\phi_{3},\left|V_{u b}\right|,\left|V_{c b}\right|\right)$ are accessible through theoretically clean measurements, which makes them the most promising probes.

\section{2. $\left|V_{u b}\right|$ measurements}

The $\left|V_{u b}\right|$ parameter can be measured through exclusive and inclusive semileptonic $B$ decays. Currently, averaged values of exclusive $\left(\left|V_{u b}^{\text {excl }}\right|=(3.67 \pm 0.09(\exp ) \pm 0.12(\right.$ theo $\left.)) \times 10^{-3}\right)$ and inclusive $\left(\left|V_{u b}^{\text {incl }}\right|=\left(4.52 \pm 0.15(\exp )_{-0.14}^{+0.11}(\right.\right.$ theo $\left.\left.)\right) \times 10^{-3}\right)\left|V_{u b}\right|$ measurements show a 4 standard deviation discrepancy [4], which could already represent a tantalizing indication of a departure from the Standard Model [5].

The most promising channel for exclusive $\left|V_{u b}\right|$ measurements at Belle II is $B \rightarrow \pi l v$. The differential branching fraction with respect to $q^{2}$, where $q$ is the four momentum imparted to the leptonic part of the final state, is proportional to $\left|V_{u b}\right|$ and to the $B \rightarrow \pi$ form factor. The form factor is a function of $q^{2}$ and is calculated by lattice QCD and LCSR [6]. Uncertainty of the form-factor predictions is currently limiting the precision of the exclusive $\left|V_{u b}\right|$ measurements, but a factor of five improvement is expected in the next decade [7], which makes a measurement of $\left|V_{u b}\right|$ particularly relevant in the next few years.

Due to the neutrino in the final state, the momentum of the $B$ meson (and hence $q^{2}$ ) is defined from the initial energy of the $e^{+} e^{-}$collision and from the momentum of the companion $B$ meson. The accompanying meson can be reconstructed ("tagged measurement") to improve the $q^{2}$ resolution for the signal meson at a cost in reconstruction efficiency, which was $0.3 \%$ for the latest Belle analysis [8]. The limiting factor here is the number of modes that are recognised by the reconstruction algorithm. The extension to a larger set of channels in Belle II allows for a factor of two better reconstruction efficiency.

The need for the reconstruction of the companion $B$ meson can be overcome by assuming that all tracks and clusters in the calorimeter are not used in the signal $B$ reconstruction (referred to as "rest of event" [ROE]) are originated from the companion meson; hence, its momentum can be obtained by summing over contributions from the ROE components ("untagged measurement"). This assumption is only approximately valid due to the extra tracks and extra clusters from beam- 
background induced interactions and secondary interactions of primary particles produced in $e^{+} e^{-}$ collisions. Such extra contributions are suppressed with multivariate classifiers based on track and cluster quality. Optimization of the track selection allows for a better separation of signal and continuum background in the final fit, which is expected to yield a $20 \%$ selection efficiency, almost twice as large of the previous Belle analysis [9]. The expected precision of the exclusive $\left|V_{u b}\right|$ determination from untagged (tagged) analysis assuming the above mentioned progress in LQCD calculations is $1.7 \%(1.3 \%)$ on the $50 \mathrm{ab}^{-1}$ dataset.

Experiments at hadron machines also put constraints on $\left|V_{u b}\right| /\left|V_{c b}\right|$ ratio measured in exclusive decays analysing of baryonic decays [10]; however, the absolute $\left|V_{u b}\right|$ measurement remains a prerogative of Belle II.

Inclusive $\left|V_{u b}\right|$ measurements are free from form-factor assumptions and the total $B \rightarrow X_{u} l v$ rate can be calculated in an Operator Product Expansion in terms of local operators, with nonperturbative corrections first appearing at $\mathscr{O}\left(1 / m_{b}^{2}\right)$, where $m_{b}$ is $b$ quark mass. However, the measurement of the full $B \rightarrow X_{u} l v$ rate is complicated by the dominant $B \rightarrow X_{c} l v$ background, which restricts the sensitivity at the edges of the kinematical region, where $B \rightarrow X_{c} l v$ decays are prohibited. Due to the experimental difficulties in the determination of the full $B \rightarrow X_{u} l v$ rate, $\left|V_{u b}\right|$ is extracted from the differential $B \rightarrow X_{u} l v$ rate in various phase space regions. For this purpose, events with one fully reconstructed tag-side $B$ meson candidate and at least one lepton track from the signal candidate are selected. The $\left|V_{u b}\right|$ value is then extracted from the fit to the differential $B \rightarrow X_{u} l v$ rates with a fit model defined from simulation. Unfortunately, the predictions of the shapes of these functions depend on the dynamics of the decaying $b$ quark, which cannot be described perturbatively and poses a limiting factor for the inclusive $\left|V_{u b}\right|$ determination. The systematic uncertainty of inclusive $\left|V_{u b}\right|$ measurements from the description of $b$-quark dynamics is dominant and might be minimised via a model-independent treatment [11], by including $B \rightarrow X_{s} \gamma$ data (the dynamic of the $b$-quark in such processes coincides with that for $B \rightarrow X_{u} l v$ at the leading order) and by constraints on non-perturbative parameters (extracted, e.g., from $B \rightarrow X_{c} l v$ ) to the global fit. The final precision of the inclusive $\left|V_{u b}\right|$ measurement will depend on the number of the measured differential ratios, but projections to the first $5 \mathrm{ab}^{-1}$ of the Belle II data with two single-differential spectra in $m_{X}$ (the invariant mass of hadronic part of the decay) and $E_{l}$ (lepton energy) of the $B \rightarrow X_{u} l v$ decay and $E_{\gamma}$ spectrum of $B \rightarrow X_{s} \gamma$ show a factor of two improvement in inclusive $\left|V_{u b}\right|$ precision with respect to the current value [7].

All measurements currently used for inclusive $\left|V_{u b}\right|$ determination come from lepton machines [4]. These measurements require reconstruction of the tag-side candidate and control over tracks belonging to the signal candidate, which is challenging to achieve in hadron collisions.

\section{3. $\phi_{3}$ from $B \rightarrow D^{(*)} K^{(*)}$}

The CKM angle $\phi_{3}(\gamma)$ can be measured from the phase difference between tree amplitudes contributing to $B \rightarrow D^{(*)} K^{(*)}$ decays. Due to the dominance of tree amplitudes, the measurement is theoretically clean; the current value of $\phi_{3}=(-109.5 \pm 5.7)^{\circ},(70.5 \pm 5.7)^{\circ}$ where the uncertainty has no theoretical component [4] (the best Belle measurement of $\phi_{3}$ has precision of $20 \%$ [12]).

Depending on the type of $D$ decay, there are several techniques to extract $\phi_{3}$. Thanks to its unbiased trigger and to sensitivity to neutrals, the most precise Belle II $\phi_{3}$ result is expected to 

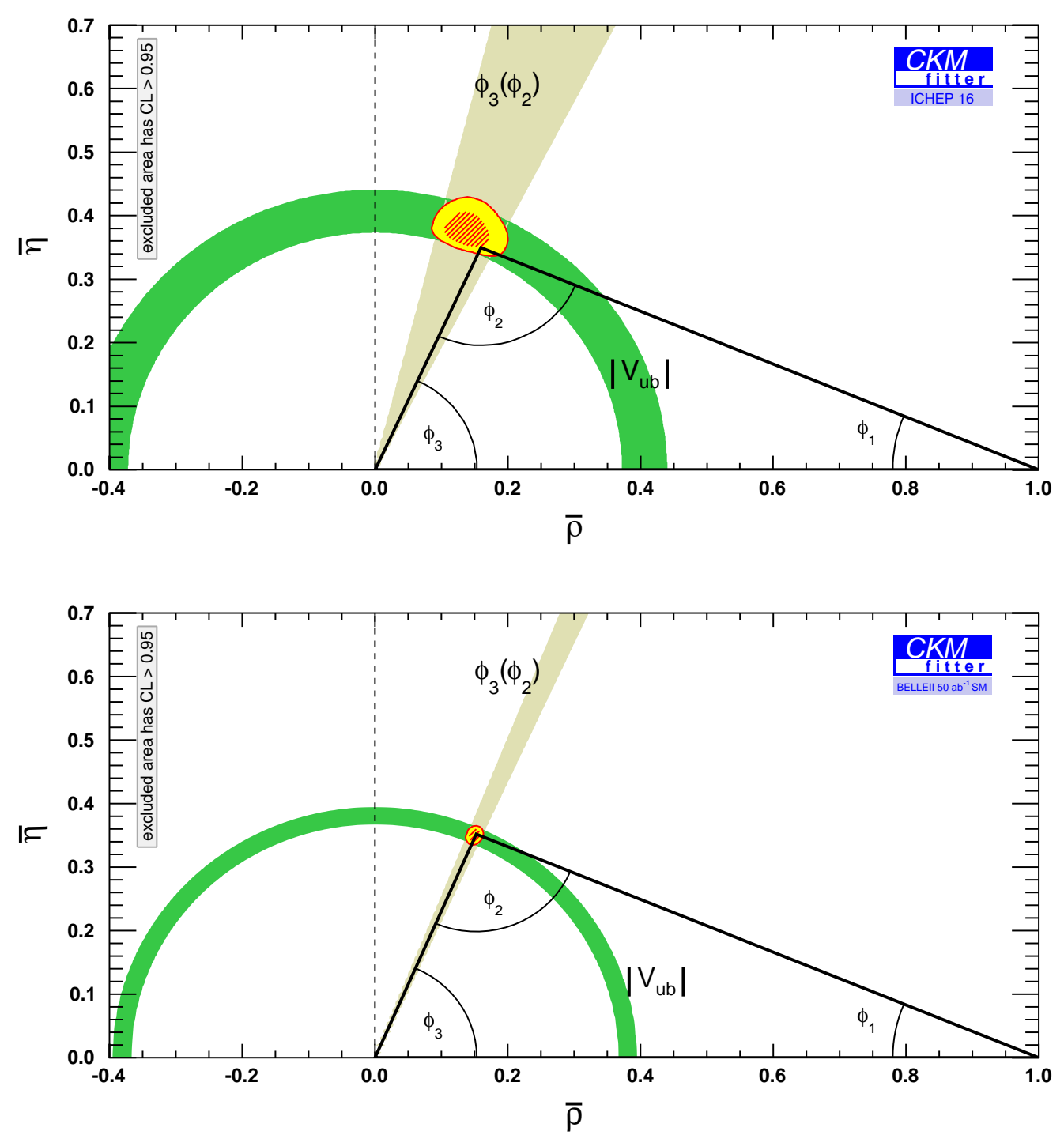

Figure 1: UT fit today (up) and extrapolated to the $50 \mathrm{ab}^{-1}$ scenario for an SM-like scenario (down) [7].

come from the Dalitz-plot analysis of self-conjugate $D$ decays [13]. The Dalitz plane is divided into a number of bins, and for each, numbers of $B^{ \pm} \rightarrow D K^{ \pm}$decays are measured. Using $D^{*}$ decays to flavour eigenstates, fractions of $D$ and $\bar{D}$ decays are also defined for each bin. Finally, amplitude-averaged strong-phase differences between $D$ and $\bar{D}$ decays are input in each bin from measurement at charm factories. Based on simulation of $B^{ \pm} \rightarrow D\left(\rightarrow K_{s}^{0} \pi \pi\right) K^{ \pm}$decays from $50 \mathrm{ab}^{-1}$ and assuming $10 \mathrm{fb}^{-1}$ collected at the $\psi(3770)$ resonance at BESIII [14], the $\phi_{3}$ precision is expected to reach $3^{\circ}$. Inclusion of results obtained from analysis of $D$ decaying to CP-eigensatates, Cabibbo-favoured and doubly Cabibbo suppressed final states, is expected to reduce the uncertainty to $1.6^{\circ}[7]$. 


\section{Conclusion}

Belle II is preparing to record $e^{+} e^{-}$collisions at the $\Upsilon(4 S)$ energy in 2018, and by 2025 it plans to have collected $50 \mathrm{ab}^{-1}$ of data. This dataset will allow improved precision measurements of currently experimentaly-limited CKM parameters, offering sharp indirect probe for the non-Standard Model. The most relevant Belle II contribution here will be the improvement in measurements of parameters accessible in tree level decays (as shown in Figure 1): the $\phi_{3}$ precision is expected to reach $1.6^{\circ}$, dominated by the model-independent Dalitz-plot analysis of $B \rightarrow D K$ decays and $\left|V_{u b}\right|$ will be measured with a precision up to $1.3 \%(3 \%)$ in exclusive (inclusive) semileptonic measurements.

\section{References}

[1] Belle-II, T. Abe et al., Belle II Technical Design Report, arXiv:1011.0352.

[2] N. Cabibbo, Unitary Symmetry and Leptonic Decays, Phys. Rev. Lett. 10 (1963) 531, [,648(1963)].

[3] M. Kobayashi and T. Maskawa, CP Violation in the Renormalizable Theory of Weak Interaction, Prog. Theor. Phys. 49 (1973) 652.

[4] Y. Amhis et al., Averages of b-hadron, c-hadron, and $\tau$-lepton properties as of summer 2016, arXiv: 1612.07233.

[5] A. Greljo, G. Isidori, and D. Marzocca, On the breaking of Lepton Flavor Universality in B decays, JHEP 07 (2015) 142, arXiv: 1506.01705.

[6] B. Melic, $B \rightarrow \pi, B_{s} \rightarrow K$ form factors and $V_{u b}$ determination, in Proceedings, 34th International Conference on High Energy Physics (ICHEP 2008): Philadelphia, Pennsylvania, July 30-August 5, 2008, 2008. arXiv:0810.1144.

[7] E. Kou, P. Urquijo, The Belle II collaboration, and The B2TiP theory community, The Belle II Physics Book, In preparation .

[8] Belle, A. Sibidanov et al., Study of Exclusive $B \rightarrow X_{u} \ell v$ Decays and Extraction of $\left\|V_{u b}\right\|$ using Full Reconstruction Tagging at the Belle Experiment, Phys. Rev. D88 (2013), no. 3 032005, arXiv:1306.2781.

[9] Belle, H. Ha et al., Measurement of the decay $B^{0} \rightarrow \pi^{-} \ell^{+} v$ and determination of $\left|V_{u b}\right|$, Phys. Rev. D83 (2011) 071101, arXiv:1012.0090.

[10] LHCb, R. Aaij et al., Determination of the quark coupling strength $\left|V_{u b}\right|$ using baryonic decays, Nature Phys. 11 (2015) 743, arXiv:1504.01568.

[11] Z. Ligeti, I. W. Stewart, and F. J. Tackmann, Treating the $b$ quark distribution function with reliable uncertainties, Phys. Rev. D78 (2008) 114014, arXiv:0807.1926.

[12] Belle, A. Poluektov et al., Evidence for direct $C P$ violation in the decay $B \rightarrow D^{(*)} K, D \rightarrow K_{s} \pi^{+} \pi^{-}$and measurement of the CKM phase $\phi_{3}$, Phys. Rev. D81 (2010) 112002, arXiv:1003.3360.

[13] A. Giri, Y. Grossman, A. Soffer, and J. Zupan, Determining gamma using $B^{ \pm} \rightarrow D K^{ \pm}$with multibody $D$ decays, Phys. Rev. D68 (2003) 054018, arXiv: hep-ph/ 0303187.

[14] D. M. Asner et al., Physics at BES-III, Int. J. Mod. Phys. A24 (2009) S1, arXiv: 0809.1869. 